\title{
Major Sources
}

Chen Zumei 陳祖美. Li Qingzhao xinzhuan 李清照新傳. Beijing: Beijing chubanshe, 2001.

Egan, Ronald. The Burden of Female Talent: The Poet Li Qingzhao and Her History in China. Cambridge, MA: Asia Center, Harvard University, 2013.

Deng Hongmei 鄧紅梅. Li Qingzhao xinzhuan 李清照新傳. Shanghai: Shanghai guji chubanshe, 2005.

Huang Shengzhang 黄盛璋. “Li Qingzhao shiji kaobian” 李清照事跡 考辨. Wenxue yanjiu 文學研究 1957.3. Reprinted in Li Qingzhao yanjiu huibian 李清照研究彙編. Ed. Zhou Kangxie 周康熇. Hong Kong: Chongwen shudian, 1974. Pp. 188-242.

. “Zhao Mingcheng, Li Qingzhao fufu nianpu” 趙明誠，李清 照夫婦年譜. Shandong shengzhi ziliao 山東省志資料 1957.3. Reprinted in Li Qingzhao yanjiu huibian 李清照研究彙編. Ed. Zhou Kangxie 周康熳. Hong Kong: Chongwen shudian, 1974. Pp. 13287.

Nangong Bo 南宫搏. Li Qingzhao de hou bansheng 李清照的後半生. Taipei: Commercial Press, 1971, reprinted in 1996.

Rexroth, Kenneth and Ling Zhong. Li Ching-chao: Complete Poems. New York: New Directions, 1979.

Wang Yingzhi 王英志. Li Qingzhao ji 李清照集. Nanjing: Fenghuang chubanshe, 2007.

Wang Zhongwen 王仲文. Li Qingzhao ji jiaozhu 李清照集校註. Beijing: Renmin wenxue chubanshe, 1979.

Xu Peijun 徐培均. Li Qingzhao ji jianzhu 李清照集箋注, rev. ed. Shanghai: Shanghai guji chubanshe, 2013.

Yu Zhonghang 于中航. Li Qingzhao nianpu 李清照年譜. Taipei: Commercial Press, 1995.

Zhuge Yibing 諸葛憶兵. Li Qingzhao shici 李清照詩詞. Beijing: Zhonghua shuju, 2013. 
